We also investigate the dynamics of tidal stripping of globular clusters in a galactic potential field with a view towards establishing the relationship between the observed limiting radii of globular clusters in our own Galaxy and their orbit shapes. We initiate a program of large-scale, self consistent numerical simulations to test the validity of the classical King tidal radius under different conditions. Our simulations, though limited in the amount of parameter space considered, reveal that slow removal of stars and tidal heating may be responsible for maintaining a halo of extratidal stars which can significantly alter the appearance of the surface density profile from the profile predicted by the classical King model. Our simulations are not of sufficient duration to determine whether this halo is eventually removed.

The final portion of this work is aimed at determining accurate limiting radii for a sample of 12 halo globulars. These clusters are interesting in that their metallicities appear to be correlated with their previously inferred orbit shapes. Deep, two-colour, photographic photometry is used to select and count stars with colours and magnitudes consistent with the cluster-specific colour-magnitude sequences. Owing to the consequent reduction in the number of contaminating foreground stars, we are able to push the star counts to significantly lower surface densities than has previously been possible. We find that several of our sample clusters show extra-tidal wings in their surface density profiles which are consistent with the results of our numerical simulations. We identify the extra-tidal material with stars which are in the process of being removed from the clusters. We argue that the extra-tidal stars limit the accuracy to which we can resolve the limiting radii of stars still bound to the clusters.

\section{DIFFRACTION EFFECTS IN MICHELSON STELLAR INTERFEROMETRY}

\author{
Michael Hyrnevych \\ Chatterton Astronomy Department, School of Physics, University \\ of Sydney, NSW 2006 \\ March 1992
}

The Sydney University Stellar Interferometer (SUSI) has very long pathlengths between the two input apertures and so diffraction modifies the phase distribution across interferring wavefronts. These two paths will, in general, be unequal and consequently diffraction will give rise to phase differences between the two wavefronts at the detectors. This difference causes a loss in the experimentally determined fringe visibility, which is used in the estimation of stellar diameters. In order to make accurate measurements of stellar diameters (to $\approx 2 \%$ or better) it is important to accurately compensate for the diffractive effects.

The calculation of this diffraction loss is complicated by the fact that we have relatively large beam diameters in comparison to the internal propagation lengths of SUSI. This leads to calculations of diffraction with relatively large Fresnel numbers. The Fresnel approximation is useful in making diffraction integrals more readily calculable and analytically tractable. However, the range of its validity (in terms of distance from the aperture) for a circular aperture is not clear. The conventional Fresnel approximation is tested against a direct numerical evaluation of the Kirchhoff diffraction integral in both intensity and phase to enable the range of validity of the approximation to be determined. A new form of the Fresnel approximation is also introduced, that amounts to a coordinate transform, which is found to be more accurate over a much larger region than the conventional form of the approximation.

Experimental verification of diffraction patterns for circular apertures have been performed in the past, but these have tended to be for low Fresnel numbers. Generally SUSI operates at somewhat higher Fresnel numbers. Previous experiments have also been generally limited to measuring the spatial features of diffraction patterns, notably the positions of diffraction rings. In this thesis we experimentally verify the use of the Fresnel approximation at quite large Fresnel numbers for both spatial features and relative intensity.

In applying this to SUSI, the diffractive effects from the optical components that starlight encounters within the instrument is considered and shown to be negligible. Finally, a calculation for visibility loss is given.

\section{RADIO EMISSION FROM ACTIVE LATE-TYPE DWARF STARS}

Jeremy Lim Jin Leong

Department of Physics and Mathematics, Macquarie University,

NSW 2019

June 1991

This thesis describes the first attempt to measure the wideband dynamic spectra (i.e. intensity as a function of frequency and time) of stellar radio flares. The observations were made at metre (200 to $500 \mathrm{MHz}$ ) and decimetre (1300 to $1800 \mathrm{MHz}$ ) wavelengths on the Parkes 64-m telescope with a 96-channel filterbank spectrometer constructed by the author. The aim of this experiment was to test for (i) plasma emission from its drift in frequency with time, and from its emission simultaneously at the fundamental and second harmonic, and (ii) electron-cyclotron maser emission which may be confined to small regions of the frequency-time domain. The experiment placed upper limits on the rate of occurrence of strong decimetre-wave flares, and similarly of strong metre-wave flares with frequency drifts similar to Type II bursts on the Sun.

This thesis also describes the first use of the Australia Telescope Compact Array to study stellar radio emission. The study of the young and rapidly-rotating K1-dwarf star AB Doradus provided the first conclusive evidence for rotational modulation of stellar radio emission. This observation suggests the existence of compact magnetic field structures in stellar coronae, and strong turbulent magnetic fields in starspots. Another highlight of the study was the discovery of a new flare star, Rositter 137B. The quiescent emission of this star is an order of magnitude brighter, and is observed more reliably, than that of any other flare star. The observation of this star also provided the first conclusive evidence for polarized quiescent emission. A model is proposed that explains both the unpolarized and polarized quiescent emission of Rositter 137B, and the puzzling constancy in the sense of polarization of flares from particular flare stars.

Finally, the spectrometer constructed by the author was used in conjunction with other spectrometers for a survey for pulsars. A total of 46 new pulsars were found in the galactic plane, and 15 recycled pulsars found in globular clusters. 